\section{PREFERENCIAS DE CONSUMO DE CHILES (Capsicum annuum L.) NATIVOS EN LOS VALLES CENTRALES DE OAXACA, MÉXICO}

\section{CONSUMPTION PREFERENCES OF PEPPER (Capsicum annuum L.) LANDRACES IN THE CENTRAL VALLEYS OF OAXACA, MÉXICO}

\section{Édgar Castellón-Martínez¹, J. Luis Chávez-Servia², José C. Carrillo-Rodríguez ${ }^{1 *}$ y Araceli M.Vera-Guzman}

${ }^{1}$ Instituto Tecnológico del Valle de Oaxaca, Ex-Hacienda Nazareno, Santa Cruz Xoxocotlán. 71230, Oaxaca, México. ${ }^{2}$ Instituto Politécnico Nacional CIIDIR-Unidad Oaxaca, Hornos \#1003. 71230, Santa Cruz Xoxocotlán, Oaxaca, México.

*Autor para correspondencia (jcarrillo_rodriguez@hotmail.com)

\section{RESUMEN}

\begin{abstract}
Las preferencias de consumo de las personas son de tipo afectivo - cognitivo con base en la percepción de estímulos, y se transforman en necesidades dentro de su contexto sociocultural y territorial. Con el objetivo de evaluar la preferencia de consumo de chiles regionales en 18 centros poblados de los Valles Centrales de Oaxaca, se aplicaron encuestas a 1287 personas mayores de 15 años. Los resultados mostraron que los entrevistados prefieren consumir chile 'Jalapeño' (37.7 \%), 'Chile de Árbol' (18.9\%), 'Chile de Agua' (15.8 \%) y 'Serrano' (13.1 \%). En referencia a los chiles regionales, los consumidores prefieren 'Chile de Agua' (67.1\%), 'Paradito' (13.8\%) y 'Tusta' (8.2\%), por su sabor y grado de picor. Las formas preferidas de consumo son: en salsa (36.5 $\%)$, relleno $(28 \%)$, asado $(21.9 \%)$ o rebanado en rajas (11.2 \%). Las familias compran desde algunos frutos $(62.3 \%)$ hasta un kilogramo $(27.3$ $\%)$ por semana de chiles locales, y existe cierta regionalización de las preferencias de consumo: hacia el sur de los Valles Centrales, además de 'Chile de Agua, se prefiere 'Tusta,' 'Solterito' y 'Tabiche', y en las zonas este, norte y centro se prefiere a 'Chile de Agua,' 'Paradito' y 'Piquín'. Los encuestados percibieron que los chiles 'Tusta' (40.1\%), 'Piquín' (37.1 \%) y 'Paradito' (14\%) son los acervos genéticos que se están perdiendo. La percepción de demanda y utilización documentada en este trabajo muestra que la cocina local puede favorecer la conservación de los chiles regionales.
\end{abstract}

Palabras clave: Capsicum spp., conocimiento local, cultura alimentaria.

\section{SUMMARY}

Consumption preferences are cognitive - affective based on stimulus perception, and they are transformed into needs within their sociocultural and territorial context. In order to evaluate the consumption preferences of regional peppers in 18 towns in the Central Valleys of Oaxaca, 1287 people over 15 years old were surveyed. The results showed that the people interviewed prefer consumption of 'Jalapeño' (37.7\%), 'Chile de Ârbol' (18.9\%), 'Chile de Agua' (15.8\%) and 'Chile Serrano' (13.13\%) peppers. In reference to regional peppers, the consumers showed major preference for 'Chile de Agua' (67.1\%), 'Solterito' (13.8\%) and 'Tusta' (8.2\%), based on their flavor and pungency. The regional peppers are consumed in sauces $(36.5 \%)$, as fillings $(28 \%)$, whole roasted $(21.9 \%)$ or in raw slices $(11.2 \%)$. Families buy anywhere from a few pods $(62.3 \%)$ to one kilogram per week $(27.3 \%)$ of the local peppers. Certain regional differences were observed in consumption preferences: in the southern zone of the Central Valleys people preferred 'Chile de Agua', 'Tusta', 'Solterito' and 'Tabiche' peppers, while in the eastern, northern and central zones 'Chile de Agua,' 'Paradito' and 'Piquín' peppers were preferred. The people surveyed believed that the gene pools of the 'Tusta' (40.1\%), 'Piquín' (37.1\%) and 'Paradito' (14.0 $\%)$ peppers are endangered. The perception of demand and utilization documented in this study shows that local cooking could enhance the conservation of regional peppers.

Index words: Capsicum spp., local knowledge, food culture.

\section{INTRODUCCIÓN}

El cultivo de chile (Capsicum spp.) en México tiene gran importancia social y económica debido a que es un producto de exportación (> 600 mil toneladas de chile verde) y a que tiene amplia distribución y un consumo cada vez más generalizado (FAOSTAT, 2009; Aguilar-Rincón et al., 2010). El consumo per capita varía entre 8 y $9 \mathrm{~kg}$, del cual $75 \%$ se consume en fresco (González, 2010). En el país se producen anualmente 1.9 millones de toneladas, y de éstas alrededor de 700 mil toneladas se destinan al comercio exterior (SIAP, 2010). En Oaxaca, en el año 2010 se reportaron 1725 ha cosechadas de chile verde: 'Habanero', 'Seco', 'Costeño, 'Pasilla, 'Jalapeño, 'Serrano, 'Chile de Agua' y 'Soledad', entre otros, y se produjeron más de 8 mil toneladas. En el mismo año, en la región de los Valles Centrales, Oaxaca, se cosecharon 249.2 ha con una producción de 1584 toneladas (OEIDRUS, 2010).

La tradición del consumo de chile en México ha perdurado desde tiempos prehispánicos y forma parte de la dieta diaria de los mexicanos, junto con los productos derivados del maíz (Zea maíz L.), calabaza (Cucurbita pepo L.), frijol (Phaseolus vulgaris L.), cacao (Theobroma cacao L.), aguacate (Persea americana Mill.), tomate (Solanum lycopersicum L.) y otros productos más, y particularmente en Oaxaca donde hay gran historia cultural culinaria (Flannery et al., 1967; Sánchez, 2006; Perry y Flannery, 2007). Los habitantes de Mesoamérica siempre han comido chile por el placer de su sabor y picor. El contacto de la capsaicina con las neuronas sensoriales da como resultado la liberación de opioides, como las endorfinas, que son sustancias que bloquean el dolor y que provocan un estado placentero. El consumo sucesivo de chile provocaría entonces una descarga mayor de estas endorfinas, de manera que el consumo de chile resultaría más placentero que el dolor que podría causar (López, 2003; Long, 2008).

La capsaicina (trans-8-metil-N-vanillil-6-nonenamida) confiere la característica picante a los frutos de chile. Este compuesto tiene actividad analgésica y antiinflamatoria, y se utiliza con fines terapéuticos para tratar dolores provocados por la artritis reumatoide y la neuropatía diabética. No obstante, los argumentos de beneficios o efectos dañinos de la capsaicina son controvertidos; algunas referencias señalan que la capsaicina es un agente carcinógeno, co-cancerígeno o promotor de tumores, mientras que otras remarcan sus 
efectos quimiopreventivos y quimioterapéuticos (Surh y Lee, 1995; Surh, 2002). Según Luo et al. (2010), los capsaicinoides (capsaicina, dihidrocapsaicina nordihidrocapsaicina, homodihidrocapsaicina, homocapsaicina, etc.) ejercen múltiples efectos fisiológicos en la salud humana por su valor potencial en la clínica de alivio del dolor, la prevención del cáncer y la pérdida de peso, que incluye la actividad de antioxidante.

La cultura del consumo de chile en México hace que se tengan percepciones o creencias diferentes acerca de sus beneficios o efectos dañinos. En el estudio de López-Carrillo et al. (1995) hecho en 240 residentes de la ciudad de México mayores de 19 años, se determinó que el chile previene o cura enfermedades (61.3\%), un bajo porcentaje (15\%) considera que es causante de alguna enfermedad y otros más que es inocuo para la salud (10\%). En relación con las preferencias, en nueve ciudades de los Estados de Coahuila, Nuevo León y Tamaulipas, a través de una encuesta a mil consumidores, Rodríguez (2005) encuentra alta preferencia por los chiles 'Jalapeño' (37.3 \%), 'Piquín' (29.6 \%) y 'Serrano' $(24.0 \%)$, mientras que otros tipos presentaron preferencias de menos de $5 \%$; en estos hallazgos no se consideran las preferencias según el sexo.

En los chiles nativos de Oaxaca ('Tusta, 'Tabaquero', 'Solterito, 'Piquín, 'Nanchita, 'Costeño’ y ‘Chile de Agua'), VeraGuzmán et al. (2011) determinaron, además de diferencias en el contenido de capsaicinoides, divergencias significativas en el contenido de flavonoides, fenoles y carotenoides. Es de esperar que el contenido de capsaicinoides y demás metabolitos secundarios influyan en el olor, sabor y picor característico de los chiles regionales, compuestos que son identificados de alguna manera por los consumidores y definen sus preferencias. En la región Valles Centrales de Oaxaca es económicamente importante la producción de chiles regionales, pero se desconocen aspectos culturales relacionados con el consumo. Así, en este trabajo el objetivo fue evaluar las preferencias y razones de consumo de los chiles regionales de los Valles Centrales de Oaxaca, con el propósito de aportar información que ayude a diseñar estrategias regionales de conservación de los acervos genéticos de Capsicum.

\section{MATERIALES Y METÓDOS}

\section{Población objetivo y tamaño de muestra}

El estudio se llevó a cabo en los Valles Centrales de Oaxaca del sureste de México, localizado entre $16^{\circ} 01^{\prime}$ y $17^{\circ} 33^{\prime}$

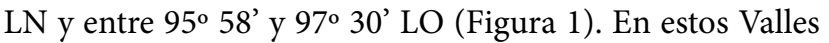
hay una población de un poco más de un millón de habitantes (47\% hombres y $53 \%$ mujeres), en 249083 hogares. Comprende los distritos geopolíticos de Etla, Zaachila, Zimatlán, Centro, Tlacolula, Ejutla, Ocotlán y Miahuatlán, y está integrada por 153 municipios (INEGI, 2005). La población objetivo está constituida por 18 centros poblados (Figura 1) con más de 10 mil habitantes. Los encuestados fueron individuos mayores de 15 años de edad.

Para definir el tamaño de muestra se utilizó como base el conteo de población y vivienda del 2005 (INEGI, 2005) y se hizo un muestreo aleatorio estratificado por municipio. El tamaño de la muestra se obtuvo mediante la fórmula propuesta por Martínez (1999) con un error muestral de $2.5 \%$ :

$$
n=\frac{N x Z^{2} x \operatorname{Px} Q}{E^{2}(N-1)+Z^{2} x \operatorname{Px} Q}=1287
$$

donde: $N=430960$ habitantes mayores de 15 años de los Valles Centrales de Oaxaca (INEGI, 2005); $P$, probabilidad de que el entrevistado sea consumidor de Capsicum =0.7; $Q$, probabilidad de que el entrevistado no fuera consumidor de Capsicum $=0.3$; E, error $\alpha=0.025$; y $Z$, constante $=1.96$. El tamaño de la muestra estimada se distribuyó de forma proporcional al número de habitantes mayores de 15 años de cada centro poblado objetivo.

\section{Características de la encuesta}

Se aplicó una encuesta personal con duración aproximada de $5 \mathrm{~min}$. Las preguntas generales se enfocaron a obtener información del sexo del encuestado y tipo de chile que más consume. En la segunda parte las preguntas específicas se orientaron a la descripción de las preferencias por los chiles regionales: chile que más prefiere, razón y forma de consumo, chile que más adquiere o compra, cantidad que consume la familia, número de integrantes de la familia que consumen chile, y la percepción sobre qué tipo o variante de chile consideran que se está erosionando. En la pregunta del chile regional que más prefiere se mostraron imágenes de los principales tipos considerados propios de la región: 'Chile de Agua, 'Piquín, 'Tusta, 'Paradito' o 'Solterito' y 'Tabiche'. Las encuestas se aplicaron en lugares con alta concentración de personas, como la Central de Abastos de la ciudad de Oaxaca, mercados municipales, y parques y tianguis de cada poblado seleccionado. En este trabajo se define preferencia a la respuesta directa del entrevistado(a) del tipo de chile que prefiere consumir sin considerar los volúmenes de consumo.

\section{Análisis estadístico}

Con las respuestas de cada entrevistado, mediante la prueba de $\chi^{2}$ se evaluó la bondad de ajuste entre las clases registradas para cada pregunta $(\mathrm{P} \leq 0.05)$. También se probaron con $\chi^{2}$ las diferencias en las respuestas entre hombres y mujeres. Para determinar la relación entre variables, se calculó la correlación de Spearman (t de Student, 0.05). En forma complementaria, se hizo un análisis de correspondencia 


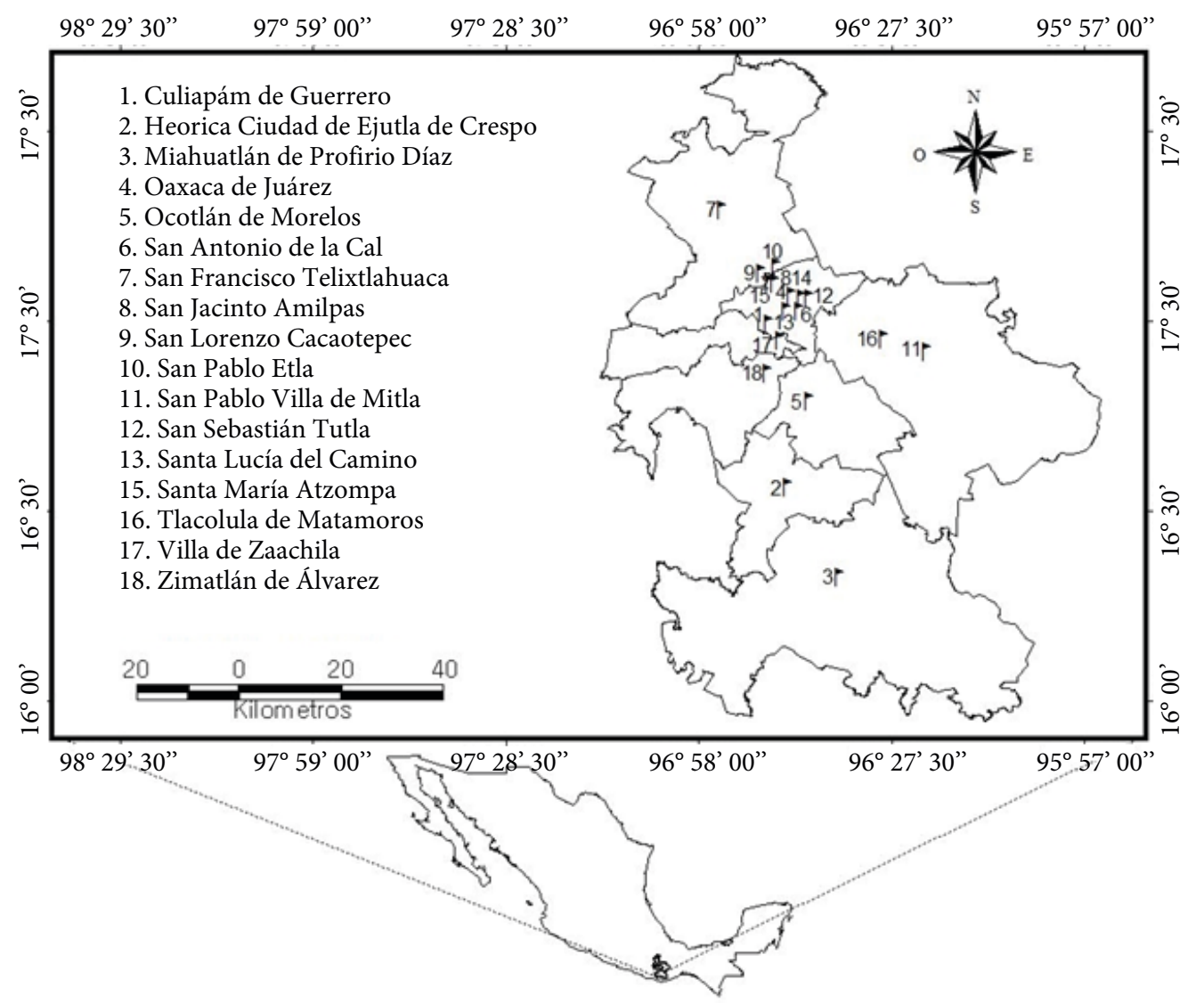

Figura 1. Centros poblados de los Valles Centrales de Oaxaca donde se aplicó la encuesta sobre preferencias y razones de consumo de chile.

múltiple para describir los patrones de preferencias entre los entrevistados y entender las razones y preferencias de consumo. Todos los análisis se hicieron con el paquete estadístico SPSS ${ }^{\circledR}$ PASW Statistics versión 18.0 para Windows.

\section{RESULTADOS Y DISCUSIÓN}

En cuanto a la variante o tipo de chile que más se consume hubo diferencias significativas entre las clases de respuestas; el 'Jalapeño' fue el más consumido (37.7 \%), seguido de 'Serrano' (13.3\%), de 'Árbol' (18.9\%) y de los regionales 'Chile de agua' (15.8 \%) y 'Piquín' (6.5 \%) (Cuadro 1). Rodríguez (2005) determinó un patrón semejante en nueve ciudades de los estados de Coahuila, Nuevo León y Tamaulipas. Esto se atribuye, en parte, a que los chiles 'Jalapeño' y 'Serrano' son los de mayor producción y comercialización en México (SIAP, 2010) y, por consiguiente, son los más conocidos. En contraposición, hay variantes o formas de frutos de chile de ámbito exclusivamente local o regional que incluso no se conoce en los estados donde son cultivados, como en este caso los tipos locales de Oaxaca.
En cuanto a preferencias según el sexo de los encuestados, se presentaron diferencias relacionadas con los tipos de chile que más se consumen, formas y razones de consumo $\left(\chi^{2}=145.7, \mathrm{P} \leq 0.01\right)$, todo en mayor proporción en las mujeres ya que hubo mayor número de encuestadas, lo que coincide con la información de INEGI (2005) de mayor proporción de mujeres $(53 \%)$ en los Valles Centrales de Oaxaca. En general, entre mujeres y entre hombres los patrones de preferencias, razones y formas de consumo son semejantes, y se repiten en todas las preguntas relacionadas con las variantes de chiles nativos. Por ejemplo, tanto en hombres como en mujeres se presentó un alto nivel de consumo de 'Chile de Agua,' 'Paradito' y 'Tusta', situación que se repite en formas de consumo, y así sucesivamente.

Entre la comunidad de entrevistados se identificó que se consumen alrededor de 15 variantes o tipos diferentes de chile de tres especies, principalmente: C. annuum, C. pubescens R. \& P. ('Canario') y C. chinense Jacq. ('Habanero'), esencialmente para consumo en fresco y algunos tanto en fresco como en seco. Entre las variantes locales se notó mayor frecuencia de consumo del 'Chile de Agua,' 'Paradito' y 
'Tusta', y menor frecuencia en 'Piquín' y 'Tabiche. Entre las razones de consumo se destaca el sabor (69.9 \%) y el picor (20\%), y estas percepciones ocurren en las tres principales formas de consumo: en salsas, relleno (p. ej., 'Chile de Agua') y asado (Cuadro 1). Por ello el chile es uno de los ingredientes esenciales en la cocina oaxaqueña (Pérez, 2004). Las preferencias de consumo parecen estar relacionadas con las percepciones del contenido de capsaicinoides, flavonoides y fenoles, entre otros compuestos, como se detectó en la evaluación de la composición de chiles regionales de Yucatán (Cázares-Sánchez et al., 2005), de Puebla (MoránBañuelos et al., 2008) y en los propios de Oaxaca evaluados por Vera-Guzmán et al. (2011).

Las razones de consumo aquí descritas coinciden con las referidas por Rodríguez (2005) en tres estados del norte de México, aunque ahí se consumen otras variantes de chile. Por ejemplo, en Oaxaca los chiles regionales que más se compran para consumo familiar son: 'Chile de Agua' (71.9 \%), 'Paradito' (12.7 \%) y 'Tusta' (7.2 \%), mientras que en el norte Rodríguez (2005) determinó que se consume más el 'Piquín. En este trabajo también se consideró al 'Piquín' dentro de la encuesta, pero no se destacó como uno de los de mayor preferencia ni adquisición por la familia (Cuadro 2), probablemente porque en los Valles Centrales de Oaxaca se conserva y aprovecha una mayor diversidad de variantes de chile que en la región norte de México, ya que varias familias oaxaqueñas de las zonas rurales cultivan sus propios chiles que consumen $(2.2 \%)$.

Los volúmenes de compra dependieron del tipo de chile que se adquiere, desde frutos individuales, por gramos o por kilogramo. En general, los resultados muestran que para la familia se compra desde algunos frutos de chile hasta $1 \mathrm{~kg}(89.6 \%)$, en un rango de 1 a 6 personas por familia (87.6\%), como se muestra en el Cuadro 2. Esto indica que, no obstante la presencia de los chiles ampliamente comercializados como 'Jalapeño' y 'Serrano', las variantes locales tienen su nicho de mercado, aunque no con altos volúmenes. Esto es parte de los factores que contribuyen a que no se pierda la diversidad de tipos locales de chile en las diferentes regiones de México, lo que favorece su conservación basada en la utilización o consumo. Este planteamiento es apoyado por los trabajos de Aguilar-Rincón et al. (2010) y Lesur (2006), en relación con la idea de que la mayor diversidad de Capsicum en México sólo es conocida y utilizada a nivel regional o local.

En los Cuadros 2 y 3 se observan patrones semejantes entre hombres y mujeres acerca de sus preferencias de compra, volúmenes de adquisición, integrantes de la familia que consumen chile y opiniones acerca de la variante de chile que está en riesgo; sin embargo, las mujeres son las que frecuentemente tienen más participación que el hombre. Por ejem- plo, tanto hombres como mujeres adquieren 'Chile de Agua' para la familia, pero las frecuencia absoluta de mujeres duplica a la de hombres, hecho que se repite en volúmenes de compra y percepción de orientas de chile en riesgo.

Respecto a cuál es el chile local o regional que se está perdiendo, los encuestados mencionaron, de acuerdo con sus percepciones, que las variantes 'Tusta,' 'Piquín' y 'Paradito' son las que están en riesgo (Cuadro 3). Este riesgo puede estar asociado con la propia dinámica de las poblaciones cultivadas o silvestres de la variante de chile; por ejemplo, 'Tusta' sólo es cultivado en la región de Miahuatlán y Sierra Sur, pero 'Piquín' y 'Paradito' son casi exclusivamente de recolección en los terrenos cercanos a las zonas de cultivo y solares (López, 2007a; Aguilar-Rincón et al., 2010).

En este trabajo se encontraron diferentes relaciones entre las preguntas (Cuadro 4). Las relaciones más significativas ocurrieron entre el chile local que más se consume con las razones y formas de consumo y el chile local que más adquiere. Esto es de relevancia cultural y conveniente para la conservación de los acervos genéticos de los chiles locales, porque indica, hasta cierto punto, una convicción en las preferencias del consumo de los chile regionales como parte de la reafirmación cultural (Pérez, 2004; Long, 2008), en este caso para la preparación de platillos regionales.Además, estos hechos están relacionados con aspectos de mercado regional o nacional; si bien el consumo y preferencia se relacionan con aspectos socioculturales locales, las influencias del mercado son determinantes en los cambios de patrones de consumo que en ocasiones no es a favor de alimentos tradicionales o de alto valor nutritivo (Pelto y Pelto, 1983).

En el análisis de correspondencia se determinó que las formas de consumo y el chile regional que más compra, fueron las respuestas de mayor valor descriptivo de la variabilidad concentrada en la dimensión 1 (abscisas, Figura 2), y las de mayor relevancia en la dimensión 2 (ordenadas, Figura 2) fueron las preferencias y razones de consumo. Así, las preferencias de consumo de chiles regionales por los encuestados siguen patrones muy locales. En la Figura 2 se muestra la dispersión de respuestas de 1287 individuos mayores de 15 años de edad, con respecto a la variante de chile que más prefieren consumir, en cuatro zonas de los Valles Centrales de Oaxaca. En las zonas Norte (Etla, Telixtlahuaca y Cacaotepec), Este (Tlacolula y Mitla) y Centro, se prefiere 'Chile de Agua', 'Solterito' y 'Piquín. Hacia la zona Sur, además de 'Chile de Agua', se observó una alta preferencia por los chiles 'Tusta' y 'Tabiche', lo que coincide con las regiones donde se producen. También, como era de esperarse que en la zona Centro ubicada alrededor de la ciudad de Oaxaca, se tiene amplia preferencia, en orden decreciente, por 'Chile de Agua,' 'Paradito, 'Piquín,' 'Tusta' y 'Tabiche. Esta regionalización de preferencias también está relacionada con las 
Cuadro 1. Frecuencias observadas entre los entrevistados en relación con las preferencias y razones de consumo de chiles en los Valles Centrales de Oaxaca, México. Febrero-mayo 2011.

\begin{tabular}{|c|c|c|c|}
\hline Preguntas y clases de respuestas & Hombre & Mujer & $\begin{array}{l}\text { Frec. obs. } \\
\text { (\% del total) }\end{array}$ \\
\hline \multicolumn{4}{|l|}{ Tipo de chile que más consume $\left(\chi^{2}=2485.25^{\star *}, n=1287\right)$} \\
\hline Jalapeño (Capsicum annuum L.) & 177 & 308 & $485(37.7)$ \\
\hline Serrano (C. annuum L.) & 57 & 114 & $171(13.3)$ \\
\hline De árbol (C. annuum L.) & 82 & 161 & $243(18.9)$ \\
\hline De agua (C. annuum L.) & 52 & 151 & $203(15.8)$ \\
\hline Piquín (C. annuum L.) & 2 & 6 & $8(0.6)$ \\
\hline Pasilla (C. annuum L.) & 28 & 56 & $84(6.5)$ \\
\hline Guajillo (C. annuum L.) & 1 & 11 & $12(0.9)$ \\
\hline Tusta (C. annuum L.) & 6 & 7 & $13(1.0)$ \\
\hline Tabiche (C. annuum L.) & 4 & 11 & $15(1.2)$ \\
\hline Paradito (C. annuum L.) & 1 & 5 & $6(0.5)$ \\
\hline Poblano (C. annuum L.) & 0 & 5 & $5(0.4)$ \\
\hline Canario (C. pubescens R. \& P.) & 11 & 18 & $29(2.2)$ \\
\hline Otros ('Habanero', 'Morita,' ‘Chilhuacle') & 6 & 7 & $13(1.0)$ \\
\hline \multicolumn{4}{|l|}{ Tipo de chile regional que más consume $\left(\chi^{2}=1811.02^{\star \star}, \mathrm{n}=1287\right)$} \\
\hline Chile de agua $\left(\chi^{2}=70.6^{\star *}, \mathrm{n}=863\right)$ & 257 & 606 & $863(67.1)$ \\
\hline Piquín $\left(\chi^{2}=0.6 n s, n=73\right)$ & 32 & 41 & $73(5.7)$ \\
\hline Tusta $\left(\chi^{2}=1.7 \mathrm{~ns}, \mathrm{n}=105\right)$ & 43 & 62 & $105(8.2)$ \\
\hline Paradito $\left(\chi^{2}=2.5 \mathrm{~ns}, \mathrm{n}=178\right)$ & 74 & 104 & $178(13.8)$ \\
\hline Tabiche $\left(\chi^{2}=5.0^{*}, \mathrm{n}=68\right)$ & 21 & 47 & $68(5.3)$ \\
\hline \multicolumn{4}{|l|}{ Razón de consumo de los chiles regionales $\left(\chi^{2}=2823.34^{\star \star}, n=1287\right)$} \\
\hline Sabor & 281 & 619 & $900(69.9)$ \\
\hline Aroma & 6 & 10 & $16(1.2)$ \\
\hline Gran picor & 118 & 140 & $258(20.0)$ \\
\hline Poco picor & 14 & 36 & $50(3.9)$ \\
\hline Multifuncionalidad en su preparación & 5 & 40 & $45(3.5)$ \\
\hline $\begin{array}{l}\text { Otro (nutricional, fácil de conseguir, jugoso, color, es el que se da, aro- } \\
\text { ma, no hace daño) }\end{array}$ & 3 & 15 & $18(1.4)$ \\
\hline \multicolumn{4}{|l|}{ Forma de consumo de chiles regionales $\left(\chi^{2}=821.25^{\star \star}, n=1287\right)$} \\
\hline Salsa $\left(\chi^{2}=21.5^{\star \star}, \mathrm{n}=470\right)$ & 164 & 306 & $470(36.5)$ \\
\hline Relleno $\left(\chi^{2}=22.3^{\star \star}, \mathrm{n}=361\right)$ & 117 & 244 & $361(28.0)$ \\
\hline En fresco $\left(\chi^{2}=0.2 \mathrm{~ns}, \mathrm{n}=27\right)$ & 15 & 12 & $27(2.1)$ \\
\hline Asado $\left(\chi^{2}=19.9^{\star *}, \mathrm{n}=282\right)$ & 88 & 194 & $282(21.9)$ \\
\hline En rajas $\left(\chi^{2}=13.3^{* *}, \mathrm{n}=144\right)$ & 41 & 103 & $144(11.2)$ \\
\hline Otro (frito) & 2 & 1 & $3(0.2)$ \\
\hline
\end{tabular}

$\mathrm{ns}=$ diferencias no significativas $(\mathrm{P}>0.05)$; ${ }^{*}$ diferencias significativas a nivel $\mathrm{P} \leq 0.05 ;{ }^{* *}$ diferencias significativas a nivel $\mathrm{P} \leq 0.01$ (prueba de $\left.\chi^{2}\right)$; Frec. obs. $=$ Frecuencia observada. 
Cuadro 2. Frecuencias observadas de preferencias de adquisición, volúmenes de compra y número de integrantes de la familia que consumen chiles regionales en los Valles Centrales de Oaxaca. Febrero-Mayo 2011.

\begin{tabular}{|c|c|c|c|}
\hline Preguntas y clases de respuestas & Hombre & Mujer & $\begin{array}{l}\text { Frec. obs. } \\
\text { (\% del total) }\end{array}$ \\
\hline \multicolumn{4}{|c|}{ Chile regional que más se adquiere para la familia $\left(\chi^{2}=1874.01^{* *}, \mathrm{n}=1098\right)$} \\
\hline Agua & 254 & 535 & 789 (71.9) \\
\hline Piquín & 19 & 15 & $34(3.1)$ \\
\hline Tusta & 35 & 44 & $79(7.2)$ \\
\hline Paradito & 59 & 81 & $140(12.7)$ \\
\hline Tabiche & 17 & 39 & $56(5.1)$ \\
\hline \multicolumn{4}{|c|}{ Cuánto compran (en g por semana) de chile por familia $\left(\chi^{2}=2546.95^{\star \star}, n=1126\right)$} \\
\hline $0-500$ & 248 & 453 & $701(62.3)$ \\
\hline $501-1000$ & 99 & 209 & $308(27.3$ \\
\hline $1001-1500$ & 4 & 25 & $29(2.6)$ \\
\hline $1501-2000$ & 27 & 30 & $57(5.1)$ \\
\hline $2501-3000$ & 10 & 10 & $20(1.8)$ \\
\hline $3500-4000$ & 3 & 1 & $4(0.4)$ \\
\hline Más de 4000 & 2 & 5 & $7(0.6)$ \\
\hline \multicolumn{4}{|c|}{ Número de integrantes de la familia que consumen algún tipo de chile $\left(\chi^{2}=728.216^{* \star}, \mathrm{n}=1123\right)$} \\
\hline 1 a 3 & 145 & 237 & $382(34.0)$ \\
\hline 4 a 6 & 209 & 393 & $602(53.6)$ \\
\hline 7 a 9 & 31 & 68 & $99(8.8)$ \\
\hline Más de 9 & 8 & 32 & $40(3.6)$ \\
\hline
\end{tabular}

${ }^{*}$ diferencias significativas a nivel $\mathrm{P} \leq 0.01\left(\right.$ prueba de $\chi^{2}$ ); Frec. obs. $=$ frecuencia observada.

Cuadro 3. Frecuencias observadas de la percepción de los entrevistados acerca de la variante de chile que se está perdiendo en los Valles Centrales de Oaxaca. Febrero-Mayo 2011.

\begin{tabular}{|c|c|c|c|}
\hline Preguntas y clases de respuestas & Hombre & Mujer & $\begin{array}{l}\text { Frec. obs. } \\
\text { (\% del total) }\end{array}$ \\
\hline \multicolumn{4}{|c|}{ Clases de respuestas $\left(\chi^{2}=1013.31^{\star \star}, \mathrm{n}=1085\right)$} \\
\hline 'Chile de agua' $\left(\chi^{2}=7.6^{\star *}, \mathrm{n}=35\right)$ & 6 & 29 & $35(3.2)$ \\
\hline 'Piquín' $\left(\chi^{2}=19.4^{* *}, \mathrm{n}=403\right)$ & 139 & 264 & $403(37.1)$ \\
\hline 'Tusta' $\left(\chi^{2}=17.4^{* *}, \mathrm{n}=435\right)$ & 156 & 279 & $435(40.1)$ \\
\hline 'Paradito' $\left(\chi^{2}=4.3^{\star}, \mathrm{n}=152\right)$ & 58 & 94 & $152(14.0)$ \\
\hline 'Tabiche' $\left(\chi^{2}=3.1 \mathrm{~ns}, \mathrm{n}=59\right)$ & 20 & 39 & $59(5.4)$ \\
\hline Otro ('Chile de Piedra’) & 1 & 0 & $1(0.1)$ \\
\hline
\end{tabular}

$\mathrm{ns}=$ no significativo $(\mathrm{P}>0.05) ;{ }^{*}$ significativo a nivel $\mathrm{P} \leq 0.05 ;{ }^{* *}$ significativo a nivel $\mathrm{P} \leq 0.01$ (prueba de $\left.\chi^{2}\right)$; Frec. obs. $=$ frecuencia observada. 
Cuadro 4. Correlaciones de ( $r$ ) entre clases de respuestas de los entrevistados en función de nueve preguntas relacionadas con preferencias y razones de consumo de chiles regionales en los Valles Centrales de Oaxaca. Febrero-Mayo 2011.

\begin{tabular}{|c|c|c|c|c|c|c|c|c|c|}
\hline Preguntas & SE & $\mathrm{CCM}$ & CRCM & $\mathrm{RC}$ & FC & CRMA & $\mathrm{CC}$ & NFCC & CRP \\
\hline Sexo del encuestado (SE) & & $0.06^{*}$ & $-0.09^{\star *}$ & -0.04 & 0.03 & $-0.08^{* *}$ & $<0.01$ & $0.07^{\star}$ & -0.04 \\
\hline Chile que más consume (CMC) & & & $0.06^{*}$ & $<0.01$ & -0.03 & 0.05 & 0.01 & 0.01 & 0.03 \\
\hline $\begin{array}{l}\text { Chile local que más consume } \\
\text { (CRMC) }\end{array}$ & & & & $0.24^{* *}$ & $-0.43^{* *}$ & $0.90^{\star *}$ & $0.07^{*}$ & 0.02 & $-0.11^{\star *}$ \\
\hline Razón de consumo (RC) & & & & & $-0.17^{\star *}$ & $0.26^{\star *}$ & $-0.08^{\star *}$ & $-0.07^{*}$ & 0.04 \\
\hline Forma de consumo (FM) & & & & & & $-0.37^{\star \star}$ & -0.03 & 0.03 & 0.02 \\
\hline $\begin{array}{l}\text { Chile local que más adquiere } \\
\text { (CRMA) }\end{array}$ & & & & & & & 0.03 & 0.01 & $-0.15^{* *}$ \\
\hline Cuánto compra (CC) & & & & & & & & $0.32^{* \star}$ & 0.03 \\
\hline $\begin{array}{l}\text { Núm. Familiares que consumen } \\
\text { chile (NFCC) }\end{array}$ & & & & & & & & & -0.01 \\
\hline Chile que se está perdiendo (CRP) & & & & & & & & & \\
\hline
\end{tabular}

${ }^{*}$ significativo al nivel $\mathrm{P} \leq 0.05$; ${ }^{* *}$ significativo al nivel $\mathrm{P} \leq 0.01$ ( $\mathrm{t}$ de Student).

zonas de distribución y producción en los Valles Centrales de Oaxaca (López, 2007a y b; Aguilar-Rincón et al., 2010).

Los resultados muestran que el 'Chile de Agua' es de alta preferencia y consumo en los Valles Centrales de Oaxaca; es decir, ya tiene cierta popularidad, como también resaltan otros investigadores (López, 2007b; Pablo et al., 2009; Aguilar-Rincón , 2010). Sin embargo, existen otras variantes de C. annuum muy regionales como 'Tusta,' 'Paradito', 'Tabiche' y 'Nanchita' que son muy conocidos en este ámbito local, de modo que su preservación y utilización, o por lo menos conocimiento de que existen, está determinado por su utilización en la cocina local. En este sentido, los consumidores alcanzan a percibir sabores y aromas diferentes en cada tipo, percepción relacionada con la cultura alimenticia (Long, 2008), sus creencias (López-Carrillo et al., 1995) y la composición de los frutos (Cázares-Sánchez et al., 2005; Moran-Bañuelos et al., 2008; Vera-Guzmán et al., 2011).

\section{CONCLUSIONES}

En los Valles Centrales de Oaxaca hay alta preferencia por el consumo de las variantes de chile 'Jalapeño', 'Chile de Árbol, 'Chile de Agua’ y 'Serrano'. Entre los chiles regionales, además del 'Chile de Agua', destacan 'Paradito’ y 'Tusta' preferidos por su sabor y grado de picor; en salsas, rellenos, asados o en rajas, son las formas preferidas de consumo. Las familias compran desde algunos frutos hasta un kilogramo por semana de los chiles locales. En cuanto a la regionalización de las preferencias de consumo de los chiles locales, hacia el sur de los Valles, además del 'Chile de Agua', prefieren 'Tusta' y 'Tabiche', y en las zonas este, norte y centro se prefiere el 'Chile de Agua,' 'Paradito' y 'Piquín. Los encuestados perciben que los chiles 'Tusta,' 'Piquín' y 'Paradito', son los acervos genéticos que se están perdiendo. 

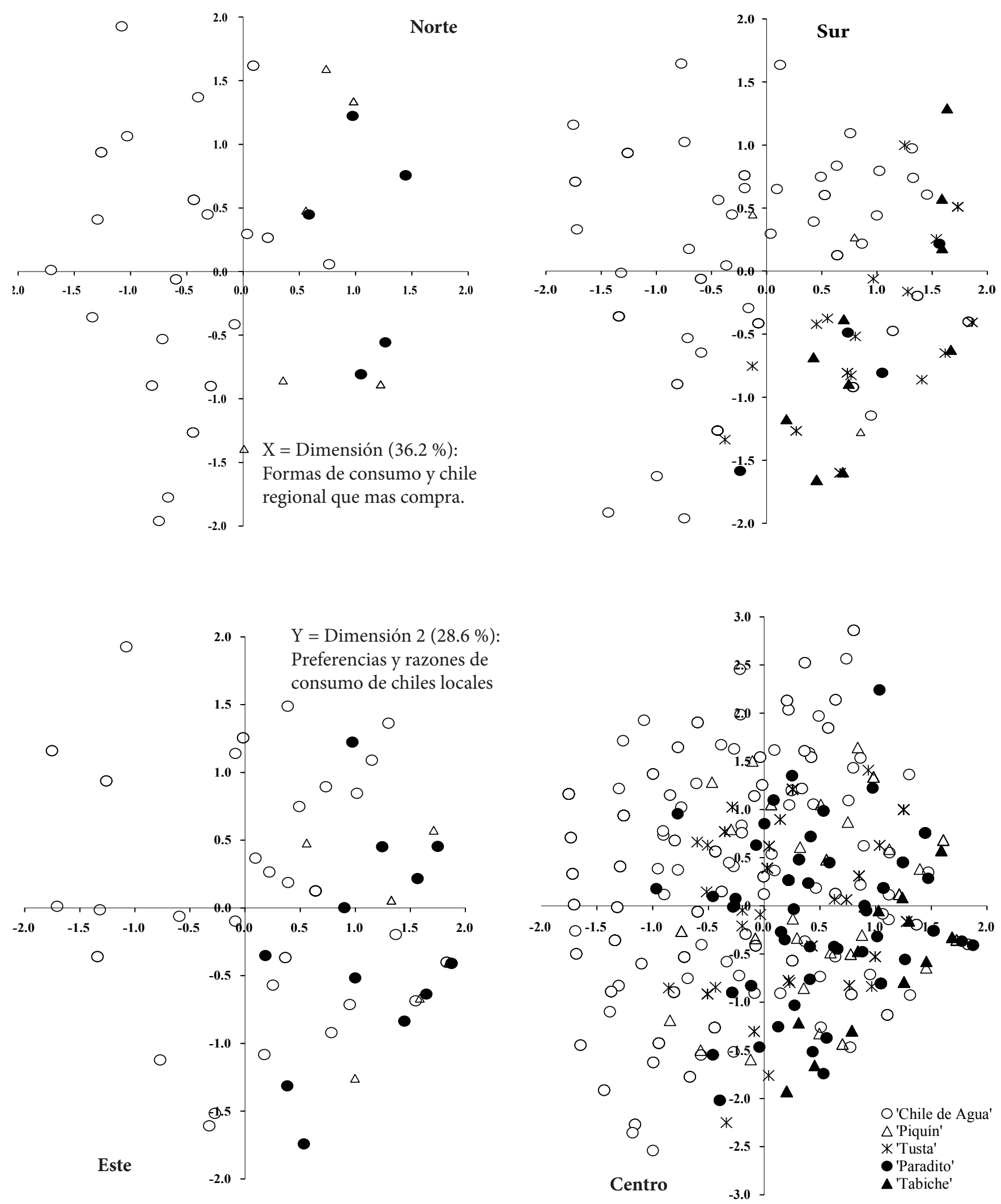

Figura 2. Dispersión de las preferencias de consumo $(n=1257)$ de chiles regionales en los Valles Centrales de Oaxaca, con base en las dos primeras dimensiones del análisis de correspondencia. Zona Norte: Etla-Telixtlahuaca; Zona Sur: OcotlánMiahuatlán; Zona Este: Mitla-Tlacolula; y Zona Centro: alrededor de la ciudad de Oaxaca. 


\section{BIBLIOGRAFÍA}

Aguilar-Rincón V H, T Corona-Torres, P López-López, L LatournerieMoreno, M. Ramírez-Meraz, H Villalón-Mendoza, J A AguilarCastillo (2010) Los Chiles de México y su Distribución. SINAREFI, Colegio de Postgraduados, INIFAP, IT-Conkal, UNAL y UAN. Montecillo, Texcoco, Estado de México. 114 p.

Cázares-Sánchez E, P Ramírez-Vallejo, F Castillo-González, M SotoHernández, T Rodríguez-González, J L Chávez-Servia (2005) Capsaicinoides y preferencia de uso en diferentes morfotipos de chile (Capsicum annuum L.) del centro-oriente de Yucatán. Agrociencia 39:627-638

FAOSTAT, Food and Agriculture Organization of the United Nations (2009) Exportaciones: País por producto. Organización de las Naciones Unidad para la Agricultura y la Alimentación (FAO). Roma, Italia. Disponible en: http://faostat.fao.org/site/ 342 /default.aspx (Diciembre 2011).

Flannery K V, A V T Kirkby, M J Kirkby, A W Williams (1967) Farming systems and political growth in ancient Oaxaca. Science 158:445-454.

González J A (2010) El cultivo del chile Capsicum annuum L. Boletín 9 Junio 2010. Secretaría de Agricultura, Ganadería, Desarrollo Rural, Pesca y Alimentación. Guanajuato, México. Disponible en: http:// www.sagarpa.gob.mx/Delegaciones/guanajuato/Lists/Boletines/ DispForm. aspx?ID=74\&RootFolder=\%2FDelegaciones\%2Fgu anajuato\%2FLists\%2FBoletines\%2F2010\%2FJunio (Diciembre 2011).

INEGI, Instituto Nacional de Estadística, Geografía e Informática (2005) II Conteo de Población y Vivienda 2005. Disponible en: http://www. inegi.org.mx (Diciembre 2010).

Lesur L (2006) Manual del Cultivo del Chile. Ed. Trillas. México, D.F. 80 p.

Long J (2008) Tecnología alimentaria prehispánica. Est. Cult. Náhualt 39:127136.

López-Carrillo L, M C Fernández-Ortega, R Costa-Díaz, J Franco-Marina, T Alejandro-Badillo (1995) Creencias sobre el consumo de chile y la salud en la ciudad de México. Salud Públ. 37:339-343.

López G O (2003) Chilli: la especia del nuevo mundo. Ciencias 69:66-75.

López P (2007a) La diversidad genética del chile (Capsicum spp.) en Oaxaca, México. Rev. Agroproduce 16:5-7.

López P (2007b) El Chile de Agua: un chile típico de los Valles Centrales de Oaxaca. Rev. Agroproduce 16:8-12.
Luo X J, J Peng, Y J Li (2010) Recent advances in the study on capsaicinoids and capsinoids. Eur. J. Pharmacol. 650:1-7.

Martínez C (1999) Estadísticas y Muestreo. Eco Ediciones. Santa Fé de Bogotá, Colombia. $886 \mathrm{p}$

Morán-Bañuelos S H, V H Aguilar-Rincón, T Corona-Torres, F CastilloGonzález, R M Soto-Hernández, R San Miguel-Chávez (2008) Capsaicinoides en chiles nativos de Puebla, México. Agrociencia 42:807-816

OEIDRUS, Oficina Estatal de Información para el Desarrollo Rural Sustentable (2010) Anuario Estadístico de la Producción Agrícola 2010, Oaxaca. Disponible en: http://www.oeidrus-portal.gob.mx/ oeidrus_oax/a1.php (Diciembre 2011).

Pablo E, J A Mejía, A Carballo, G García, V H Aguilar, T Corona. (2009) Calidad de semilla en colectas de chile de agua (Capsicum annuum L.) de los Valles Centrales, Oaxaca, México. Agric. Téc. Méx. 35:257-266.

Pelto G, P Pelto (1983) Diet and delocalization: dietary change since 1750. J. Interdisc. History 14:507-528.

Pérez E (2004) Recetario Mixe de Oaxaca. Consejo Nacional para la Cultura y las Artes, México, D.F. 155 p.

Perry L, K V Flannery (2007) Precolumbian use of chili peppers in the Valley of Oaxaca, Mexico. Proc. Nat. Acad. Sci. USA 104:11905-11909.

Rodríguez L A (2005) Preferencia del consumidor por el chile piquín en comparación con otros chiles en el noreste de México. Rev. Chapingo S. Hort. 11:279-281.

SIAP, Sistema de Información Agroalimentaria y Pesquera (2010) Anuario Estadístico de la Producción Agrícola 2010. Sistema de Información Agroalimentaria y Pesquera de la Secretaria de Agricultura, Ganadería, Desarrollo Rural, Pesca y Alimentación. Disponible en: http://www.siap.gob.mx/index.php?option=com wrapper\&view=wrapper\&Itemid $=350$ (Diciembre 2011)

Sánchez A V (2006) La fiesta del gusto: La construcción de México a través de sus comidas. Opción 22:9-25.

Surh Y J (2002) More than spice: capsaicin in hot chili peppers makes tumor cells commit suicide. J. Nat. Cancer Inst. 94:1263-1265.

Surh Y J, S S Lee (1995) Capsaicin in hot chili pepper: Carcinogen, co-carcinogen or anticarcinogen? Food Chem. Toxicol. 34:313-316.

Vera-Guzmán A M, J L Chávez-Servia, J C Carrillo-Rodríguez, M G López (2011) Phytochemical evaluation of wild and cultivated pepper (Capsicum annuum L. and C. pubescens Ruiz \& Pav.) from Oaxaca, Mexico. Chilean J. Agric. Res. 71:578-585. 